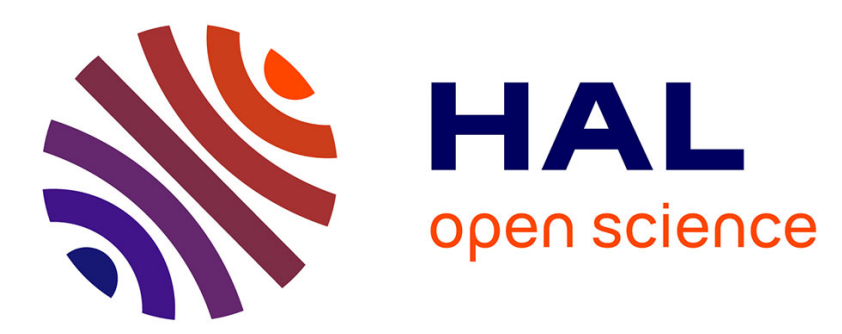

\title{
Interval observers for linear time-invariant systems with disturbances
}

\author{
Frédéric Mazenc, Olivier Bernard
}

\section{To cite this version:}

Frédéric Mazenc, Olivier Bernard. Interval observers for linear time-invariant systems with disturbances. Automatica, 2011, 47 (1), pp.140-147. 10.1016/j.automatica.2010.10.019 . hal-00555464

\section{HAL Id: hal-00555464 \\ https://hal.science/hal-00555464}

Submitted on 25 Jan 2011

HAL is a multi-disciplinary open access archive for the deposit and dissemination of scientific research documents, whether they are published or not. The documents may come from teaching and research institutions in France or abroad, or from public or private research centers.
L'archive ouverte pluridisciplinaire HAL, est destinée au dépôt et à la diffusion de documents scientifiques de niveau recherche, publiés ou non, émanant des établissements d'enseignement et de recherche français ou étrangers, des laboratoires publics ou privés. 


\title{
Interval observers for linear time-invariant systems with disturbances *
}

\author{
Frédéric Mazenc ${ }^{\mathrm{a}}$, Olivier Bernard ${ }^{\mathrm{b}}$,

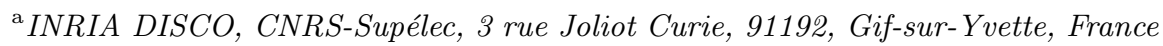 \\ ${ }^{\mathrm{b}}$ INRIA COMORE, INRIA Sophia-Antipolis, 2004 route des Lucioles, BP 93, 06902 Sophia-Antipolis, France.
}

\begin{abstract}
It is shown that, for any time-invariant exponentially stable linear system with additive disturbances, time-varying exponentially stable interval observers can be constructed. The technique of construction relies on the Jordan canonical form that any real matrix admits and on time-varying changes of coordinates for elementary Jordan blocks which lead to cooperative linear systems. The approach is applied to detectable linear systems.
\end{abstract}

Key words: Interval observer, linear system, exponential stability.

\section{Introduction}

Interval observers, appeared in the last decade as an alternative technique for robust state estimation [Gouzé et al., 2000], [Rapport et al., 2003]. It is based on differential inequalities that allow to derive bounds for the solutions of a differential system at any time instant. This approach is significantly different from the technique based on classical observers (see for instance the contributions [Luenberger, 1964], [Besançon and Hammouri, 1996], [Andrieu et al., 2009]): when only the initial condition is assumed to be unknown, both classical observers and interval observers converge asymptotically to the solutions of the considered system but, in addition, interval observers supply certain information at any instant, provided that bounds on the initial conditions are known.

Interval observers have been introduced to cope with uncertainties that are known to characterize some classes of systems. That is one of the reasons why this technique is becoming more and more popular [Moisan et al., 2009], [Raissi et al., 2005], [Mazenc and Bernard, 2010], [Mazenc et al.], and has been especially successful in the domain of

\footnotetext{
^ Corresponding author F. Mazenc. Tel.+ 33-6-07-04-23-52

Email addresses: Frederic.MAZENC@lss.supelec.fr (Frédéric Mazenc), olivier.bernard@inria.fr (Olivier Bernard).
}

biotechnological processes [Bernard and Gouzé, 2004], [Moisan and Bernard, 2005], [Moisan, 2007] where the uncertainties are large.

Interval observers can be straightforwardly constructed for any asymptotically stable cooperative systems (i.e. for systems whose Jacobian matrices have non-negative off-diagonal entries [Gouzé et al., 2000]). Indeed, these systems have the distinctive property to keep the partial order between two trajectories [Smith, 1995]. Thus, if the (unknown) initial condition of the real system can be bounded between two known values, the trajectories of the same system starting from these bounds will enclose the real trajectory. Observe that cooperative continuous-time linear systems are always positive systems but this is not the case for discrete-time linear systems. These systems are positive systems if and only if the system matrices are nonnegative (see [Haddad et al., 2010] and [Germani et al., 2007]). However, cooperativity is a rather specific feature, and most of the systems are not cooperative. This is the main limitation of the interval observer theory. In the present paper, we overcome it for a fundamental family of systems: the linear time-invariant exponentially stable systems with additive disturbances. We succeed in doing this by showing how any of these systems can be transformed through a change of coordinates into a cooperative system. This question has been recently solved for linear systems of dimension two in the work [Mazenc and Bernard, 2010] where two key results are 
established. First, it is proved that for a specific class of systems no time-invariant changes of coordinates can turn these systems into cooperative systems. Second, it is shown that any planar exponentially stable time-invariant linear system can be transformed into a cooperative exponentially stable linear system through a time-varying change of coordinates.

The purpose of this paper is to extend the main construction proposed for two dimensional systems in [Mazenc and Bernard, 2010]: we present a technique of construction of interval observers which applies to any linear exponentially stable time-invariant system of arbitrary finite dimension.

Our design relies on the fact that any real matrix can be transformed into a matrix of the Jordan canonical form (see [Perko, 1991, Section 1.8]). This property enables us to find changes of coordinates which transform any time-invariant exponentially stable linear system into a cooperative and exponentially stable system. Surprisingly, although the changes of coordinates we use are time-varying, the transformed systems are timeinvariant. However, the interval observers we obtain are time-varying because they involve a time-varying change of coordinates, and thus they give lower and upper bounds for the state of the system studied which depend on the time. It is worth noticing that we focus our attention only on exponentially stable linear systems because a system which is not asymptotically stable never admits open-loop interval observers. Hence our main result offers a complete picture of what can be done or not for linear systems: it implies that a timeinvariant linear system admits an interval observer if and only if it is exponentially stable.

We illustrate the broad applicability of our approach, by combining it (for observable systems) with a Luenberger observer [Luenberger, 1964], to end-up with a real-time estimate of the Luenberger observer accuracy.

The paper is organized as follows. Section 2 gives definitions and a basic result. In Section 3.1, we state that any time-invariant exponentially stable linear system can be transformed into a block diagonal system with cooperative and exponentially stable subsystems. The result of Section 3.1 is proved in Section 3.2. The main result of the paper is given and illustrated through an example in Section 4. The result is then applied to systems endowed with a Luenberger observer in Section 5.1. An illustration of this interval Luenberger observer in large dimension is presented in Section 5.2 and its efficiency is assessed with simulations. Concluding remarks are drawn in Section 6.

\section{Classical definitions and results}

\subsection{Notations and definitions}

- Any $k \times n$ matrix, whose entries are all 0 is simply denoted 0 .

- All the inequalities must be understood component by component (partial order of $\left.\mathbb{R}^{r}\right)$ i.e. $\mho_{a}=\overline{\left(\mho_{a 1}, \ldots, \mho_{a r}\right)^{\top}} \in$ $\overline{\mathbb{R}^{r} \text { and } \mho_{b}}=\left(\mho_{b 1}, \ldots, \mho_{b r}\right)^{\top} \in \mathbb{R}^{r}$ are such that $\mho_{a} \leq \mho_{b}$ if and only if, for all $i \in\{1, \ldots, r\}, \mho_{a i} \leq \mho_{b i}$.

- $\max (A, B)$ for two matrices $A=\left(a_{i j}\right)$ and $B=\left(b_{i j}\right)$ of same dimension is the matrix where each entry is $m_{i j}=\max \left(a_{i j}, b_{i j}\right)$.

- A square matrix is said to be cooperative or Metzler if its off-diagonal entries are nonnegative.

- A system $\dot{x}=f(x, w)$ is said to be cooperative if the Jacobian matrix of $f$ with respect to $x$, denoted $\frac{\partial f}{\partial x}(x, w)$, is a cooperative matrix for all $(x, w)$.

- The notations will be simplified whenever no confusion can arise from the context.

\subsection{Interval observer: definition and basic result}

For the sake of generality, we first introduce a general definition of interval observer for time-varying nonlinear systems.

Definition 1 Consider a system

$$
\dot{x}=F(t, x, w(t)),
$$

with $x \in \mathbb{R}^{n}, w \in \mathbb{R}^{\ell}$, and with $F$ of class $C^{1}$. The uncertainties $w$ are Lipschitz continuous and such that there exist two known bounds $\bar{w}(t)=\left(w^{+}(t), w^{-}(t)\right) \in$ $\mathbb{R}^{2 \ell}$, Lipschitz continuous, and such that, for all $t \geq 0$,

$$
w^{-}(t) \leq w(t) \leq w^{+}(t) .
$$

Moreover, the initial condition $x(0)=x_{0}$ is assumed to be bounded by two known bounds:

$$
x_{0}^{-} \leq x_{0} \leq x_{0}^{+} .
$$

Then, the dynamical system

$$
\dot{Z}=\varphi(t, Z, \bar{w}(t))
$$

associated with the initial condition $Z_{0}=G\left(t_{0}, x_{0}^{+}, x_{0}^{-}\right) \in$ $\mathbb{R}^{n_{z}}$ and bounds for the solution $x$ :

$$
x^{+}=H^{+}(t, Z), x^{-}=H^{-}(t, Z)
$$


(with $\varphi, H^{+}, H^{-}, G$ Lipschitz continuous of appropriate dimension), is called an interval observer (resp. an exponentially stable interval observer) for (1) if

(i) system (4) is globally uniformly asymptotically stable (resp. globally uniformly exponentially stable) when $\bar{w}$ is identically equal to zero,

(ii) for all Lipschitz continuous function $\bar{w}(t)$, all the solutions of (4) are defined over $[0,+\infty)$,

(iii) for any vectors $x_{0}, x_{0}^{-}$and $x_{0}^{+}$in $\mathbb{R}^{n}$ satisfying (3), the solutions of (1), (4) with respectively $x_{0}, Z_{0}=$ $G\left(t_{0}, x_{0}{ }^{+}, x_{0}{ }^{-}\right)$as initial condition at $t=t_{0}$, denoted respectively $x(t), Z(t)$, are defined for all $t \geq t_{0}$ and satisfy, for all $t \geq t_{0}$, the inequalities

$$
x^{-}(t)=H^{-}(t, Z(t)) \leq x(t) \leq H^{+}(t, Z(t))=x^{+}(t) .
$$

In the sequel we focus on linear systems. We recall a classical result for cooperative linear systems. For these systems the design of interval observers is straightforward.

Theorem 1 Consider the system

$$
\dot{x}=\mathcal{A} x+w(t),
$$

with $x \in \mathbb{R}^{n}, w \in \mathbb{R}^{n}$, where $\mathcal{A} \in \mathbb{R}^{n \times n}$ is a cooperative, Hurwitz and constant matrix and let $w$ be a Lipschitz continuous function bounded by two known Lipschitz continuous functions: for all $t \geq 0, w^{-}(t) \leq w(t) \leq w^{+}(t)$. The system (7) admits the following exponentially stable interval observer

$$
\begin{aligned}
\dot{z}^{+} & =\mathcal{A} z^{+}+w^{+}(t), \\
\dot{z}^{-} & =\mathcal{A} z^{-}+w^{-}(t), \\
x^{+} & =H^{+}(t, Z)=z^{+}, \\
x^{-} & =H^{-}(t, Z)=z^{-}, \\
z^{+}\left(t_{0}\right) & =G^{+}\left(t_{0}, x_{0}^{+}, x_{0}^{-}\right)=x_{0}^{+}, \\
z^{-}\left(t_{0}\right) & =G^{-}\left(t_{0}, x_{0}^{+}, x_{0}^{-}\right)=x_{0}^{-},
\end{aligned}
$$

with $Z=\left(z^{+}, z^{-}\right)^{\top}$ and $G=\left(G^{+}, G^{-}\right)^{\top}$.

The proof of this result is in [Gouzé et al., 2000]; it is directly related to the property of cooperative systems to keep the partial order between two trajectories.

\section{Transformations of linear systems into coop- erative systems}

In this section, we state and prove that any exponentially stable time-invariant linear system can be transformed into a cooperative and exponentially stable timeinvariant system through a linear time-varying change of coordinates. We will use this result extensively in the next section to construct interval observers.

\subsection{Change of coordinates: main result}

The main result of the section is the following:

Theorem 2 Consider the system

$$
\dot{x}=A x,
$$

with $x \in \mathbb{R}^{n}$, where $A \in \mathbb{R}^{n \times n}$ is a constant Hurwitz matrix. Then there exists a time-varying change of coordinates $\xi=P(t) x$, where $P$ is a matrix function whose entries are functions of class $C^{\infty}$ bounded in norm, which transforms (9) into a cooperative and exponentially stable time-invariant linear system.

\subsection{Proof of Theorem 2}

The proof splits up into three steps. First we recall that any real matrix admits a real Jordan canonical form. In the second step, we transform, through a time-varying change of coordinates, systems with an elementary Jordan form into cooperative systems. In the last stage, we combine the results of the first two parts to end the proof.

Step 1: Jordan canonical forms.

From [Perko, 1991, Section 1.8], (see also [Hsu, 2005], p.73), we deduce that there exist two integers $r \in$ $\{0,1, \ldots, n\}, s \in\{0,1, \ldots, n-1\}$ and a linear timeinvariant change of coordinates

$$
Y=\mathcal{P} x
$$

which transforms (9) into

$$
\dot{Y}=J Y,
$$

with

$$
J=\left[\begin{array}{cccc}
J_{1} & 0 & \ldots & 0 \\
0 & J_{2} & \ddots & \vdots \\
\vdots & \ddots & \ddots & 0 \\
0 & \ldots & 0 & J_{s}
\end{array}\right] \in \mathbb{R}^{n \times n}
$$

where the matrices $J_{i}$ are partitioned into two groups: the first $r$ matrices are associated with the $r$ real eigenvalues of multiplicity $n_{i}$ of $A$ and the others are associated with the imaginary eigenvalues of multiplicity $m_{i}$ of $A$. Therefore $n=\sum_{i=1}^{r} n_{i}+\sum_{r+1}^{s} 2 m_{i}$ and, for $i=1$ to $r$,

$$
J_{i}=\left[\begin{array}{rrrl}
-\mu_{i} & 1 & \ldots & 0 \\
0 & -\mu_{i} & \ddots & \vdots \\
\vdots & \ddots & \ddots & 1 \\
0 & \ldots & 0 & -\mu_{i}
\end{array}\right] \in \mathbb{R}^{n_{i} \times n_{i}}
$$


where the $\mu_{i}$ 's are positive real numbers and, for $i=r+1$ to $s$,

$$
J_{i}=\left[\begin{array}{ccccc}
\Lambda_{i} & I_{2} & 0 & \ldots & 0 \\
0 & \Lambda_{i} & I_{2} & \ddots & \vdots \\
\vdots & \ddots & \ddots & \ddots & 0 \\
\vdots & & \ddots & \ddots & I_{2} \\
0 & \ldots & \ldots & 0 & \Lambda_{i}
\end{array}\right] \in \mathbb{R}^{2 m_{i} \times 2 m_{i}}
$$

with

$$
\Lambda_{i}=\left[\begin{array}{cc}
-\kappa_{i} & \omega_{i} \\
-\omega_{i} & -\kappa_{i}
\end{array}\right] \in \mathbb{R}^{2 \times 2}
$$

and

$$
I_{2}=\left[\begin{array}{ll}
1 & 0 \\
0 & 1
\end{array}\right],
$$

where the $\kappa_{i}$ 's are positive real numbers and the $\omega_{i}$ 's are non-zero real numbers. Notice that the fact that the constants $\mu_{i}$ and $\kappa_{i}$ are positive is a consequence of the fact that $A$ is Hurwitz. Notice also that if $A$ has no real eigenvalues, then $r=0$ and if all of the eigenvalues of $A$ are real, then $n=\sum_{i=1}^{r} n_{i}$. For $j=1$ to $r$, the matrices $J_{j}$ are cooperative, but for $j=r+1$ to $s$, they are not and one can prove (see [Mazenc and Bernard, 2010]) that, in the general case, the corresponding systems $\dot{Y}_{j}=J_{j} Y_{j}$ cannot be transformed into cooperative systems through a linear time-invariant change of coordinates. On the basis of the result proposed in [Mazenc and Bernard, 2010] for planar systems, we show below that these systems can be transformed into cooperative systems through a linear time-varying change of coordinates.

\section{Step 2: time-varying change of coordinates.}

We consider the system

$$
\dot{x}=\Gamma x,
$$

with $x \in \mathbb{R}^{2 p}$ where

$$
\Gamma=\left[\begin{array}{ccccc}
\Lambda & I_{2} & 0 & \ldots & 0 \\
0 & \Lambda & I_{2} & \ddots & \vdots \\
\vdots & \ddots & \ddots & \ddots & 0 \\
\vdots & & \ddots & \ddots & I_{2} \\
0 & \ldots & \ldots & 0 & \Lambda
\end{array}\right] \in \mathbb{R}^{2 p \times 2 p}
$$

and

$$
\Lambda=\left[\begin{array}{cc}
-\kappa & \omega \\
-\omega & -\kappa
\end{array}\right] \in \mathbb{R}^{2 \times 2}
$$

with $\kappa>0, \omega \neq 0$. We state and prove the following result:

Lemma 1 The time-varying change of coordinates

$$
\varrho=\mathcal{N}(t) x
$$

with

$$
\mathcal{N}(t)=\left[\begin{array}{rrrl}
\varpi(t) & 0 & \ldots & 0 \\
0 & \varpi(t) & \ddots & \vdots \\
\vdots & \ddots & \ddots & 0 \\
0 & \ldots & 0 & \varpi(t)
\end{array}\right] \in \mathbb{R}^{2 p \times 2 p}
$$

with

$$
\varpi(t)=\left[\begin{array}{cc}
\cos (\omega t) & -\sin (\omega t) \\
\sin (\omega t) & \cos (\omega t)
\end{array}\right] \in \mathbb{R}^{2 \times 2},
$$

transforms system (17) into

$$
\dot{\varrho}=\mathcal{M} \varrho
$$

with

$$
\mathcal{M}=\left[\begin{array}{rrrrl}
-\kappa I_{2} & I_{2} & 0 & \ldots & 0 \\
0 & -\kappa I_{2} & \ddots & \ddots & \vdots \\
\vdots & \ddots & \ddots & \ddots & 0 \\
\vdots & & \ddots & \ddots & I_{2} \\
0 & \ldots & \ldots & 0 & -\kappa I_{2}
\end{array}\right] \in \mathbb{R}^{2 p \times 2 p}
$$

Proof. Let us rewrite system (17) as follows

$$
\left\{\begin{aligned}
\dot{\alpha}_{1} & =-\kappa \alpha_{1}+\omega \beta_{1}+\alpha_{2}, \\
\dot{\beta}_{1} & =-\omega \alpha_{1}-\kappa \beta_{1}+\beta_{2}, \\
& \vdots \\
\dot{\alpha}_{p-1} & =-\kappa \alpha_{p-1}+\omega \beta_{p-1}+\alpha_{p}, \\
\dot{\beta}_{p-1} & =-\omega \alpha_{p-1}-\kappa \beta_{p-1}+\beta_{p} \\
\dot{\alpha}_{p} & =-\kappa \alpha_{p}+\omega \beta_{p} \\
\dot{\beta}_{p} & =-\omega \alpha_{p}-\kappa \beta_{p} .
\end{aligned}\right.
$$

For each $\left(\alpha_{i}, \beta_{i}\right)$-subsystem, we perform the change of coordinates

$$
\left(\begin{array}{c}
a_{i} \\
b_{i}
\end{array}\right)=\varpi(t)\left(\begin{array}{c}
\alpha_{i} \\
\beta_{i}
\end{array}\right)
$$


It gives

$$
\begin{aligned}
\dot{a}_{i}= & -\omega\left[\sin (\omega t) \alpha_{i}+\cos (\omega t) \beta_{i}\right]+\cos (\omega t) \dot{\alpha}_{i} \\
& -\sin (\omega t) \dot{\beta}_{i}, \\
\dot{b}_{i}= & \omega\left[\cos (\omega t) \alpha_{i}-\sin (\omega t) \beta_{i}\right]+\sin (\omega t) \dot{\alpha}_{i} \\
& +\cos (\omega t) \dot{\beta}_{i} .
\end{aligned}
$$

It follows that, for $i \in\{1, \ldots, p-1\}$,

$$
\begin{aligned}
\dot{a}_{i}= & -\omega\left[\sin (\omega t) \alpha_{i}+\cos (\omega t) \beta_{i}\right] \\
& +\cos (\omega t)\left[-\kappa \alpha_{i}+\omega \beta_{i}+\alpha_{i+1}\right] \\
& -\sin (\omega t)\left[-\omega \alpha_{i}-\kappa \beta_{i}+\beta_{i+1}\right], \\
\dot{b}_{i}=\omega & \left.\omega \cos (\omega t) \alpha_{i}-\sin (\omega t) \beta_{i}\right] \\
& +\sin (\omega t)\left[-\kappa \alpha_{i}+\omega \beta_{i}+\alpha_{i+1}\right] \\
& +\cos (\omega t)\left[-\omega \alpha_{i}-\kappa \beta_{i}+\beta_{i+1}\right] .
\end{aligned}
$$

Therefore, for $i \in\{1, \ldots, p-1\}$,

$$
\begin{aligned}
\dot{a}_{i}= & -\kappa \cos (\omega t) \alpha_{i}+\cos (\omega t) \alpha_{i+1} \\
& +\kappa \sin (\omega t) \beta_{i}-\sin (\omega t) \beta_{i+1}, \\
\dot{b}_{i}= & -\kappa \sin (\omega t) \alpha_{i}+\sin (\omega t) \alpha_{i+1} \\
& -\kappa \cos (\omega t) \beta_{i}+\cos (\omega t) \beta_{i+1} .
\end{aligned}
$$

Thus, we obtain, for $i \in\{1, \ldots, p-1\}$,

$$
\begin{aligned}
& \dot{a}_{i}=-\kappa a_{i}+a_{i+1}, \\
& \dot{b}_{i}=-\kappa b_{i}+b_{i+1} .
\end{aligned}
$$

Similarly, we obtain

$$
\begin{aligned}
& \dot{a}_{p}=-\kappa a_{p} \\
& \dot{b}_{p}=-\kappa b_{p} .
\end{aligned}
$$

We deduce that the change of coordinates (20) transforms system (25) into system (23). This completes the proof of the Lemma 1.

Step 3: end of the proof of Theorem 2.

Thanks to the structure of matrix $J$ defined in (12), we can decompose system (11) as follows:

$$
\begin{gathered}
\dot{Y}_{a}=G Y_{a}, \\
\dot{Y}_{b, j}=J_{j} Y_{b, j}, j=r+1, \ldots, s,
\end{gathered}
$$

with

$$
G=\left[\begin{array}{cccc}
J_{1} & 0 & \ldots & 0 \\
0 & J_{2} & \ddots & \vdots \\
\vdots & \ddots & \ddots & 0 \\
0 & \ldots & 0 & J_{r}
\end{array}\right] \in \mathbb{R}^{q \times q}
$$

with $q=\sum_{i=1}^{r} n_{i}$ and $J_{j}$ defined in (14), (15), for $j=r+1$ to $s$. Lemma 1 applies to each $Y_{b, j}$-subsystem because $\omega_{j} \neq 0$. This leads us to perform the following timevarying change of coordinates

$$
\xi=\eta(t)\left(\begin{array}{c}
Y_{a} \\
Y_{b, r+1} \\
\vdots \\
Y_{b, s}
\end{array}\right)
$$

with

$$
\eta(t)=\left[\begin{array}{cccl}
I_{q} & 0 & \ldots & 0 \\
0 & \mathcal{N}_{r+1}(t) & \ddots & \vdots \\
\vdots & \ddots & \ddots & 0 \\
0 & \ldots & 0 & \mathcal{N}_{s}(t)
\end{array}\right] \in \mathbb{R}^{n \times n}
$$

where $I_{q}$ is the identity matrix of $\mathbb{R}^{q \times q}$ and, for $j=r+1$ to $s$,

$$
\mathcal{N}_{j}(t)=\left[\begin{array}{cccc}
\varpi_{j}(t) & 0 & \ldots & 0 \\
0 & \varpi_{j}(t) & \ddots & \vdots \\
\vdots & \ddots & \ddots & 0 \\
0 & \ldots & 0 & \varpi_{j}(t)
\end{array}\right] \in \mathbb{R}^{2 m_{j} \times 2 m_{j}}
$$

with

$$
\varpi_{j}(t)=\left[\begin{array}{cc}
\cos \left(\omega_{j} t\right) & -\sin \left(\omega_{j} t\right) \\
\sin \left(\omega_{j} t\right) & \cos \left(\omega_{j} t\right)
\end{array}\right] \in \mathbb{R}^{2 \times 2} .
$$

From Lemma 1, we infer that (35) transforms system (32), (33) into the system

$$
\dot{\xi}=\aleph \xi,
$$


with

$$
\aleph=\left[\begin{array}{cccc}
G & 0 & \ldots & 0 \\
0 & \mathcal{M}_{r+1} & \ddots & \vdots \\
\vdots & \ddots & \ddots & 0 \\
0 & \ldots & 0 & \mathcal{M}_{s}
\end{array}\right] \in \mathbb{R}^{n \times n}
$$

with, for $\ell=r+1$ to $s$,

$$
\mathcal{M}_{\ell}=\left[\begin{array}{ccccc}
-\kappa_{\ell} I_{2} & I_{2} & 0 & \ldots & 0 \\
0 & -\kappa_{\ell} I_{2} & I_{2} & \ddots & \vdots \\
\vdots & \ddots & \ddots & \ddots & 0 \\
\vdots & & \ddots & \ddots & I_{2} \\
0 & \ldots & \ldots & 0 & -\kappa_{\ell} I_{2}
\end{array}\right] \in \mathbb{R}^{2 m_{\ell} \times 2 m_{\ell}}
$$

Since the matrices $\mathcal{M}_{\ell}$ and $G$ are cooperative and Hurwitz (see (13) and (41)), we deduce that system (39) is cooperative and exponentially stable. We can thus apply Theorem 1 to system (39). We can conclude by observing that system (39) is obtained from (9) by performing the change of coordinates

$$
\xi=P(t) x
$$

with $P(t)=\eta(t) \mathcal{P}$, where $\mathcal{P}$ is the matrix in (10) and $\eta(t)$ is defined in (36).

\section{Interval observers for linear exponentially stable systems}

We can state and prove the main result of our work.

\subsection{Statement of the main result}

Theorem 3 Consider the system

$$
\dot{x}=A x+w(t)
$$

with $x \in \mathbb{R}^{n}, w(t) \in \mathbb{R}^{n}$, where $A \in \mathbb{R}^{n \times n}$ is a constant Hurwitz matrix and let $w$ be a Lipschitz continuous function bounded by two known Lipschitz continuous functions: for all $t \geq 0, w^{-}(t) \leq w(t) \leq w^{+}(t)$. Then there exists $P: \mathbb{R} \rightarrow \overline{\mathbb{R}}^{n \times n}$, of class $C^{\infty}$, bounded in norm and such that, for all $t \in \mathbb{R}, P(t)$ is invertible, and a cooperative, Hurwitz and constant matrix $\aleph \in \mathbb{R}^{n \times n}$ such that, for all $t \in \mathbb{R}, \dot{P}(t)=\aleph P(t)-P(t) A$. Moreover,

$$
\begin{aligned}
\dot{z}^{+} & =\aleph z^{+}+P^{+}(t) w^{+}(t)-P^{-}(t) w^{-}(t), \\
\dot{z}^{-} & =\aleph z^{-}+P^{+}(t) w^{-}(t)-P^{-}(t) w^{+}(t), \\
x^{+} & =H^{+}(t, Z)=M^{+}(t) z^{+}-M^{-}(t) z^{-}, \\
x^{-} & =H^{-}(t, Z)=M^{+}(t) z^{-}-M^{-}(t) z^{+}, \\
z^{+}\left(t_{0}\right) & =G^{+}\left(t_{0}, x_{0}^{+}, x_{0}^{-}\right) \\
& =P^{+}\left(t_{0}\right) x_{0}^{+}-P^{-}\left(t_{0}\right) x_{0}^{-}, \\
z^{-}\left(t_{0}\right) & =G^{-}\left(t_{0}, x_{0}^{+}, x_{0}^{-}\right) \\
& =P^{+}\left(t_{0}\right) x_{0}^{-}-P^{-}\left(t_{0}\right) x_{0}^{+}, \\
Z & =\left(z^{+}, z^{-}\right),
\end{aligned}
$$

where $P^{+}(t)=\max (0, P(t)), P^{-}(t)=P^{+}(t)-P(t)$ and the matrix $M(t)$ is the inverse of $P(t), M^{+}(t)=$ $\max (0, M(t))$ and $M^{-}(t)=M^{+}(t)-M(t)$, is an exponentially stable interval observer for system (43).

Remark. Theorem 2 applies to system (43) in the absence of the disturbance $w$ and leads to a system to which Theorem 1 applies. However, since there is a disturbance in system (43), we cannot directly derive an interval observer for this system from these two theorems.

\subsection{Proof of Theorem 3}

Using the remark above, we observe that Theorem 2 provides a constant, Hurwitz and cooperative matrix $\aleph$ and a function $P$ of class $C^{\infty}$, bounded in norm, such that, for all $t \in \mathbb{R}, \dot{P}(t)=\aleph P(t)-P(t) A$ and $P(t)$ is invertible. Next, we introduce the time-varying change of coordinates $z=P(t) x$, where all the entries of $P$ are functions of class $C^{\infty}$ bounded in norm, which transforms system (43) into

$$
\dot{z}=\aleph z+P(t) w(t) .
$$

We now prove that (44) is an exponentially stable interval observer for (43). The exponential stability of (44) when both $w^{+}$and $w^{-}$are identically equal to zero is a consequence of the fact that $\aleph$ is Hurwitz. Moreover the solutions of $(44)$ are defined over $[0,+\infty)$ for Lipschitz continuous functions $w^{+}, w^{-}$, because $P^{+}$and $P^{-}$are Lipschitz continuous.

Let $t_{0}$ be a nonnegative real number. Consider a solution $(x(t), Z(t))$ of (43) and (44) with initial conditions $x\left(t_{0}\right)$, $Z\left(t_{0}\right)=\left(z^{+}\left(t_{0}\right), z^{-}\left(t_{0}\right)\right)$ with

$$
\begin{aligned}
& z^{+}\left(t_{0}\right)=P^{+}\left(t_{0}\right) x_{0}^{+}-P^{-}\left(t_{0}\right) x_{0}^{-}, \\
& z^{-}\left(t_{0}\right)=P^{+}\left(t_{0}\right) x_{0}^{-}-P^{-}\left(t_{0}\right) x_{0}^{+},
\end{aligned}
$$

where $x_{0}^{+}, x_{0}^{-}$are vectors such that $x_{0}^{-} \leq x\left(t_{0}\right) \leq x_{0}^{+}$. 
We show that $z\left(t_{0}\right)=P\left(t_{0}\right) x\left(t_{0}\right)$ can be bounded, using both the equality $z\left(t_{0}\right)=\left(P^{+}\left(t_{0}\right)-P^{-}\left(t_{0}\right)\right) x\left(t_{0}\right)$, and the following inequalities:

$$
\begin{aligned}
& P^{+}\left(t_{0}\right) x_{0}^{-} \leq P^{+}\left(t_{0}\right) x\left(t_{0}\right) \leq P^{+}\left(t_{0}\right) x_{0}^{+} \\
& P^{-}\left(t_{0}\right) x_{0}^{-} \leq P^{-}\left(t_{0}\right) x\left(t_{0}\right) \leq P^{-}\left(t_{0}\right) x_{0}^{+}
\end{aligned}
$$

which hold because the entries of $P^{+}(t)$ and $P^{-}(t)$ are nonnegative. We get thus:

$$
\begin{aligned}
& P^{+}\left(t_{0}\right) x_{0}^{-}-P^{-}\left(t_{0}\right) x_{0}^{+} \leq z\left(t_{0}\right) \\
& z\left(t_{0}\right) \leq P^{+}\left(t_{0}\right) x_{0}^{+}-P^{-}\left(t_{0}\right) x_{0}^{-},
\end{aligned}
$$

or, equivalently,

$$
z^{-}\left(t_{0}\right) \leq z\left(t_{0}\right) \leq z^{+}\left(t_{0}\right) .
$$

Moreover, for all $t \geq 0$,

$$
\begin{aligned}
& P^{+}(t) w^{-}(t) \leq P^{+}(t) w(t) \leq P^{+}(t) w^{+}(t) \\
& P^{-}(t) w^{-}(t) \leq P^{-}(t) w(t) \leq P^{-}(t) w^{+}(t) .
\end{aligned}
$$

It follows that, for all $t \geq 0$,

$$
\begin{gathered}
P^{+}(t) w^{-}(t)-P^{-}(t) w^{+}(t) \leq P(t) w(t) \\
P(t) w(t) \leq P^{+}(t) w^{+}(t)-P^{-}(t) w^{-}(t) .
\end{gathered}
$$

Then $z(t)=P(t) x(t)$ is solution of (45) and since $\aleph$ is cooperative and the inequalities (48) are satisfied, we deduce from Theorem 1 that for all $t \geq t_{0}$, the inequalities

$$
z^{-}(t) \leq P(t) x(t) \leq z^{+}(t)
$$

hold. Since the entries of $M^{+}(t)$ and $M^{-}(t)$ are nonnegative, it follows that, for all $t \geq t_{0}$,

$$
\begin{aligned}
& M^{+}(t) z^{-}(t) \leq M^{+}(t) P(t) x(t) \leq M^{+}(t) z^{+}(t), \\
& M^{-}(t) z^{-}(t) \leq M^{-}(t) P(t) x(t) \leq M^{-}(t) z^{+}(t) .
\end{aligned}
$$

Since, for all $t \geq t_{0}, M^{+}(t)-M^{-}(t)=P(t)^{-1}$, it follows that, for all $t \geq t_{0}$,

$$
\begin{gathered}
M^{+}(t) z^{-}(t)-M^{-}(t) z^{+}(t) \leq x(t) \\
x(t) \leq M^{+}(t) z^{+}(t)-M^{-}(t) z^{-}(t) .
\end{gathered}
$$

This concludes the proof.

\subsection{Three dimensional example}

We illustrate our technique by constructing step by step an interval observer for the system

$$
\dot{x}=A x+w(t)
$$

with

$$
A=\frac{1}{3}\left[\begin{array}{ccc}
-1 & 0 & -4 \\
6 & -9 & 0 \\
10 & 0 & -5
\end{array}\right] .
$$

This system is not cooperative. One can check that $A$ is Hurwitz and therefore Theorem 3 applies to (55).

Step 1: Transformation of $A$ into a matrix of Jordan form.

For the matrix

$$
\mathcal{P}=\frac{1}{3}\left[\begin{array}{ccc}
1 & -3 & 1 \\
1 & 0 & 1 \\
2 & 0 & -1
\end{array}\right]
$$

the equality

$$
\mathcal{P} A \mathcal{P}^{-1}=J
$$

with

$$
J=\left[\begin{array}{ccc}
-3 & 0 & 0 \\
0 & -1 & 2 \\
0 & -2 & -1
\end{array}\right]
$$

is satisfied. The Jordan matrix $J$ is not cooperative.

Step 2: Construction of a diagonalizing time-varying change of coordinates.

For the particular case we consider, the matrix $\eta(t)$ in (36) is

$$
\eta(t)=\left[\begin{array}{ccc}
1 & 0 & 0 \\
0 & C(t) & -S(t) \\
0 & S(t) & C(t)
\end{array}\right]
$$

with $S(t)=\sin (2 t), C(t)=\cos (2 t)$ and the matrix $P(t)=\eta(t) \mathcal{P}$ is

$$
P(t)=\frac{1}{3}\left[\begin{array}{ccc}
1 & -3 & 1 \\
C(t)-2 S(t) & 0 & C(t)+S(t) \\
2 C(t)+S(t) & 0 & S(t)-C(t)
\end{array}\right] .
$$

It is solution of

$$
\dot{P}(t)=\aleph P(t)-P(t) A
$$

with

$$
\aleph=\frac{1}{3}\left[\begin{array}{ccc}
-3 & 0 & 0 \\
0 & -1 & 0 \\
0 & 0 & -1
\end{array}\right]
$$


Observe for later use that

$$
\begin{aligned}
M(t) & =P^{-1}(t) \\
& =\left[\begin{array}{ccc}
0 & C(t)-S(t) & C(t)+S(t) \\
-1 & C(t) & S(t) \\
0 & 2 C(t)+S(t) & 2 S(t)-C(t)
\end{array}\right] .
\end{aligned}
$$

Step 3: Interval observer.

According to Theorem 3, system (55) admits the following interval observer

$$
\begin{aligned}
\dot{z}^{+} & =\aleph z^{+}+P^{+}(t) w^{+}(t)-P^{-}(t) w^{-}(t), \\
\dot{z}^{-} & =\aleph z^{-}+P^{+}(t) w^{-}(t)-P^{-}(t) w^{+}(t), \\
x^{+} & =M^{+}(t) z^{+}-M^{-}(t) z^{-}, \\
x^{-} & =M^{+}(t) z^{-}-M^{-}(t) z^{+}, \\
z^{+}\left(t_{0}\right) & =P^{+}\left(t_{0}\right) x_{0}^{+}-P^{-}\left(t_{0}\right) x_{0}^{-}, \\
z^{-}\left(t_{0}\right) & =P^{+}\left(t_{0}\right) x_{0}^{-}-P^{-}\left(t_{0}\right) x_{0}^{+}, \\
Z & =\left(z^{+}, z^{-}\right),
\end{aligned}
$$

with $\aleph$ defined in (63),

$$
\begin{gathered}
P^{+}(t)=\frac{1}{3}\left[\begin{array}{ccc}
1 & 0 & 1 \\
\mathcal{L}(C(t)-2 S(t)) & 0 & \mathcal{L}(C(t)+S(t)) \\
\mathcal{L}(2 C(t)+S(t)) & 0 & \mathcal{L}(S(t)-C(t))
\end{array}\right] \\
P^{-}(t)=P^{+}(t)-P(t) \\
M^{+}(t)=\left[\begin{array}{ccc}
0 & \mathcal{L}(C(t)-S(t)) & \mathcal{L}(C(t)+S(t)) \\
0 & \mathcal{L}(C(t)) & \mathcal{L}(S(t)) \\
0 & \mathcal{L} 2 C(t)+S(t)) & \mathcal{L}(2 S(t)-C(t))
\end{array}\right]
\end{gathered}
$$

with $\mathcal{L}$ defined by $\mathcal{L}(s)=\max \{s, 0\}$ and

$$
M^{-}(t)=M^{+}(t)-M(t) .
$$

\section{Application to systems endowed with Luen- berger observers}

\subsection{Interval observers for detectable systems}

In this section, we show how interval observers and classical observers can be used simultaneously to obtain complementary information on the solutions of a linear detectable system.

We consider a system

$$
\dot{X}=\alpha X+\beta u+\phi_{1}(t)
$$

with $X \in \mathbb{R}^{n}$, where $u \in \mathbb{R}^{p}$ is an input, $\phi_{1}$ is an unknown locally Lipschitz function and $\alpha$ and $\beta$ are constant matrices. We assume that system (70) is endowed with an output

$$
y(t)=\gamma X(t)+\phi_{2}(t)
$$

where $\gamma \in \mathbb{R}^{q \times n}$ is a constant matrix such that the pair $(\alpha, \gamma)$ is detectable and $\phi_{2}$ is an unknown locally Lipschitz function. It is worth observing that we do not assume that $\alpha$ is Hurwitz.

The detectability property of the pair $(\alpha, \gamma)$ ensures that there exists a constant matrix $L \in \mathbb{R}^{n \times q}$ such that the matrix $\alpha-L \gamma$ is Hurwitz [Brogan, 1990]. Then we consider the observer

$$
\dot{\hat{X}}=\alpha \hat{X}+\beta u+L(y-\gamma \hat{X})
$$

The error variable $\tilde{X}=X-\hat{X}$ gives the error equation

$$
\dot{\tilde{X}}=(\alpha-L \gamma) \tilde{X}+\phi_{1}(t)-L \phi_{2}(t)
$$

Since $\alpha-L \gamma$ is Hurwitz, Theorem 3 applies to (73) (with the notations of Theorem 3 , we have $A=\alpha-L \gamma$, $\left.w=\phi_{1}-L \phi_{2}\right)$. Therefore an interval observer can be constructed for this system. Thanks to it, an estimate of the distance between a solution of (70) and a solution of (72) can be obtained. Indeed, if (73) admits the interval observer

$$
\dot{Z}=\varphi(t, Z, \bar{w}(t)),
$$

associated with $Z_{0}=G\left(t_{0}, \tilde{X}_{0}^{+}, \tilde{X}_{0}^{-}\right) \in \mathbb{R}^{n_{z}}$ and $\tilde{X}^{+}=$ $H^{+}(t, Z), \tilde{X}^{-}=H^{-}(t, Z)$ then, if for $t_{0} \geq 0$, the solutions $X(t), \hat{X}(t)$ satisfy

$$
\tilde{X}_{0}^{-} \leq X\left(t_{0}\right)-\hat{X}\left(t_{0}\right) \leq \tilde{X}_{0}^{+}
$$

and, for all $t \geq t_{0}, w^{-}(t) \leq w(t) \leq w^{+}(t)$, then, the solution of (74) $Z(t)$ such that $Z\left(t_{0}\right)=Z_{0}$ is such that, for all $t \geq t_{0}$,

$$
H^{-}(t, Z(t)) \leq X(t)-\hat{X}(t) \leq H^{+}(t, Z(t)) .
$$

\subsection{Interval observer design and simulation}

In this simulation example we illustrate the result of Section 5.1. In order to demonstrate the applicability of our approach to high dimensional systems, we have considered a simulation example in dimension six. Our purpose is to illustrate how the proposed design can be applied to a standard Luenberger observer to estimate, in real time, its convergence despite uncertainties (here, noise in the measurements). 


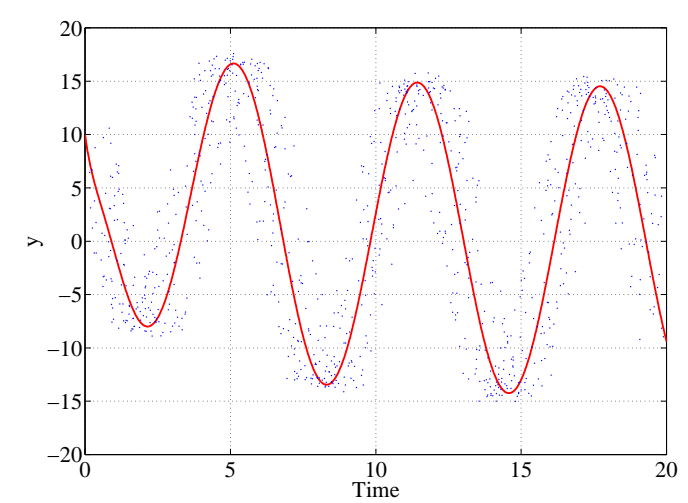

Fig. 1. Variable $x_{1}$ is supposed to be measured with a biased noise of average -0.5 .

The example we consider is the following linear system: $\dot{x}=\alpha x+\beta u(t)$ with $u(t)=\sin (t), \beta=$ $(-18,-13,-5,-4,-10,22)^{\top}$ and

$$
\alpha=\left(\begin{array}{cccccc}
-1 & 1 & 0 & 0 & -1 & 0 \\
-1 & -2 & 0 & -1 & 0 & 1 \\
-2 & 0 & -3 & -2 & 0 & 0 \\
-1 & 0 & -2 & -3 & 0 & 1 \\
-1 & 0 & 2 & 0 & -4 & 0 \\
-1 & -1 & 0 & 1 & 0 & -1
\end{array}\right)
$$

Matrix $\alpha$ has stable poles. Four of them have an imaginary part different from zero. We denote them $\omega_{1} \pm j \kappa_{1}$ and $\omega_{2} \pm j \kappa_{2}$ and $\mu_{5}$ and $\mu_{6}$ the two real eigenvalues.

We assume that the output $y=x_{1}+\phi_{2}(t)$ is available, at discrete times (every 0.05 hours) i.e. $x_{1}$ is measured, with a bounded noise : $-2 \leq \phi_{2}(t) \leq 1$, for all $t \geq 0$ (see Figure 1). The considered noise for simulation is based on a uniformly distributed noise in the interval $[-2,1]$ at the sampling instants. Function $\phi_{2}(t)$ thus results from linear interpolation of $\phi_{2}\left(t_{i}\right)$ between two sampling time instants.

Through routine calculations, one can prove that the pair $(\alpha, \gamma)$ with $\gamma=\left(\begin{array}{llll}1 & 0 & \ldots & 0\end{array}\right)$ is observable. With a pole placement algorithm, we select a matrix $K$ so that the poles of $\alpha-K \gamma$ give a desired observer closed-loop dynamics. More specially, the slowest pole of $\alpha$ being $\mu_{6}=-0.26$, we select $K$ in order to move this pole to -1 , keeping the five other poles unchanged [Brogan, 1990]. Thus, the observer dynamics is faster than the original system and the gains are, roughly speaking, not too large in order to limit observer over reactivity in the presence of large noises.

Then, the interval observer presented in Theorem 3 can be derived. Due to the dimension of the system, this in-
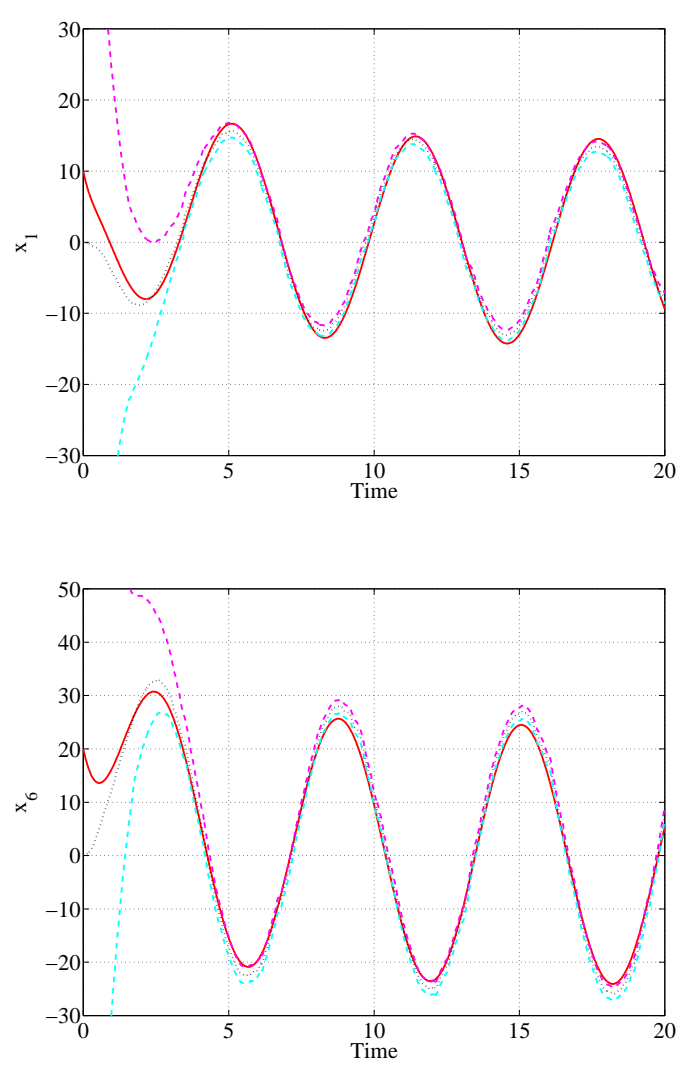

Fig. 2. Estimation of the bounds associated with the Luenberger observer (dotted lines) for the variables $x_{1}$ and $x_{6}$, and comparison with the real state (continuous line). Bounds given by the interval observer are represented by dashed lines

terval observer is of dimension twelve and given by complicated expressions. A trajectory with $t_{0}=0, x_{0}=$ $(20,10,6,20,30,40)^{\top}$ as initial condition is simulated. We also draw the solutions of the interval observer associated with unknown initial conditions but with components belonging to the interval $[0,40]$.

Figure 2 shows the estimates for this interval observer. Due to the uncertainty in the knowledge of the initial condition and to the initial change of variables, the interval is initially quite large. However its length becomes rapidly reasonably small despite the uncertainties on the measurements $y$. At the end, this approach provides an evaluation of the Luenberger observer estimation accuracy, i.e. an error interval associated to the Luenberger observer predictions. It is worth noting that we have considered a case of biased noise (the average value of the noise is -0.5) to highlight the generality of the situations where this approach applies.

\section{Conclusions}

We have presented a construction of exponentially stable interval observers for the fundamental family of 
the time-invariant exponentially stable linear systems with additive disturbances. This approach has several possible applications. One of them is the possibility to on-line assess the accuracy of classical observers such as Luenberger observers. These techniques can be improved by considering simultaneously several admissible changes of variables and by taking the intersection of all the predicted intervals [Moisan et al., 2009], [Bernard and Gouzé, 2004]. This may be important when one wants to improve the performances through bundles of interval observers in the spirit of what is done in [Moisan et al., 2009]. This will be the subject of further investigations. Besides, extensions to time-varying, nonlinear and time-delay systems are expected.

\section{References}

[Andrieu et al., 2009] V. Andrieu , L. Praly , A. Astolfi, High gain observers with updated gain and homogeneous correction terms, Automatica, vol. 45 no.2, pp. 422-428, Feb., 2009

[Bernard and Gouzé, 2004] O. Bernard, J.-L. Gouzé, Closed loop observers bundle for uncertain biotechnological models, Journal of Process Control, vol. 14, pp. 765-774, 2004.

[Besançon and Hammouri, 1996] G. Besançon, H. Hammouri, On uniform observation of nonuniformly observable systems. Systems and control letters, vol. 29, no.1, pp. 9-19, 1996.

[Brogan, 1990] W.L. Brogan, Modern Control Theory (3rd Edition), Englewood Cliffs, N.J.: Prentice Hall, 1990.

[Germani et al., 2007] A. Germani, C. Manes, P. Palumbo, State space representation of a class of MIMO systems via positive systems. Proc. of the 46th IEEE Conference on decision and control, New Orleans, LA, USA pp. 476-481, 2007.

[Gouzé et al., 2000] J.-L. Gouzé, A. Rapaport, Z. Hadj-Sadok, Interval observers for uncertain biological systems. Ecological Modelling, 133, pp. 45-56, 2000.

[Haddad et al., 2010] W. M. Haddad, V. Chellaboina, Qing Hui, Nonnegative and Compartmental Dynamical Systems. Princeton University Press, Princeton and Oxford, 2010.

[Hsu, 2005] S.B. Hsu, Ordinary differential equations with applications. Series on Applied Mathematics, Volume 16, 2005.

[Luenberger, 1964] D. Luenberger, Observing the state of a linear system. IEEE Trans. Military Electronics, vol. MIL-8, pp. 74-80, 1964

[Mazenc and Bernard, 2010] F. Mazenc, O. Bernard, Asymptotically Stable Interval Observers for Planar systems with Complex Poles. IEEE Trans. on Aut. Control, vol. 55, Issue 2, Feb. 2010, pp. 523-527.

[Mazenc et al.] F. Mazenc, S.I. Niculescu, O. Bernard, Interval Observers for Linear Systems with Delay. 48th IEEE Conference on decision and control, Shanghai, China 2009.

[Moisan, 2007] M. Moisan, Synthèse d'Observateurs par Intervalles pour des Systèmes Biologiques Mals Connus. (In French and English), Phd Thesis, Sophia-Antipolis, Sept. 2007.

[Moisan and Bernard, 2005] M. Moisan, O. Bernard, Interval Observers for non-monotone systems. Application to bioprocess models. Proc. of the 16th IFAC World Congress, Prague, Czech Republic, 2005.
[Moisan et al., 2009] M. Moisan, O. Bernard, J.-L. Gouzé, Near optimal interval observers bundle for uncertain bioreactors, Automatica, vol. 45, pp. 291-295, 2009.

[Perko, 1991] L. Perko, Differential Equations and Dynamical systems. Text in Applied Mathematics, Springer-Verlag, 1991.

[Raissi et al., 2005] T. Raissi, N. Ramdani, Y. Candau, Bounded error moving horizon state estimation for non-linear continuous time systems: application to a bioprocess system. Journal of Process Control 15, pp. 537-545, 2005.

[Rapport et al., 2003] A. Rapaport, J.-L. Gouzé, Parallelotopic and practical observers for nonlinear uncertain systems, Int. Journal. Control, vol. 76, no. 3, pp. 237-251, 2003.

[Smith, 1995] H.L. Smith, Monotone dynamical systems: An introduction to the theory of competitive and cooperative systems, American Mathematical Society., vol. 75, 1995. 